\title{
INTEGRATION IN LOCALLY COMPACT SPACES II
}

\author{
EDWIN HEWITT and H. S. ZUCKERMAN
}

§1. Introduction. The general problem of producing concrete representations for continuous linear functionals on normed linear spaces, i.e., of identifying conjugate spaces, has of course attracted the attention of many mathematicians during the last five decades and has been solved in many cases [1, pp. 59-72]. Likewise, the problem of extending a linear functional defined on a linear subspace of a normed linear space may be regarded as solved by the Hahn-Banach theorem $[1$, p. 28$]$, although problems involving "natural" extensions, like that yielding the Lebesgue integral from the Riemann integral, remain. In the present paper, we shall consider two "natural" methods of extending a certain linear functional and show that they are in fact identical. As a by-product, we obtain a concrete representation both for the original functional and for its. "natural" extension. In subsequent communications, the writers will consider topologies in certain families of linear functionals, canonical resolutions of linear functionals, and other extension problems.

§ 2. Statement of the problem. Let $X$, throughout the present paper, denote an arbitrary but fixed locally compact (= locally bicompact) Hausdorff space. Let $\underset{\infty \infty}{(s}(X, R)$ denote the linear space of all continuous real-valued functions $c(x)$ on $X$ for each of which there exists a compact subset $A$ of $X$ such that $c(x)=0$ for $X \in A^{\prime}$. For brevity, we denote by the symbol $\mathbb{C}_{+}$the set of all non-negative functions in $\underset{\infty \infty \infty}{\mathbb{S}}(X, R)$. For all subsets $Q$ of $X$, we denote by iQ the characteristic function of $Q$, and for all real-valued functions $\psi$ on $X$, we denote by $\psi_{Q}$ the function $\psi \cdot \iota_{Q}$.

The family of all compact subsets of $X$ is denoted by $a$, and the family of all closed subsets of $X$ by $\mathcal{F}$. The smallest family of sets containing $a$ and closed under the formation of countable unions, and differences, is denoted by $\bar{a}$; the smallest family of sets containing $\mathscr{F}$ and closed under the formation of countable unions and complements is denoted by $\overline{\mathscr{F}} .(\overline{\mathcal{F}}$ is of course the family of Borel sets, in the classical sense, while $\bar{a}$ is the family of Borel sets as defined by Halmos $[5$, p. 219].)

Received January 8, 1951. 
We shall be concerned throughout the present paper with a fixed (but arbitrary) positive linear functional $M$ defined on $\underset{\infty \infty \infty}{(}(X, R)$. No boundedness or other continuity condition is assumed.

In the sequel, we consider all functionals, integrals, and measurability conditions only for functions on $X$ which are non-negative. This is justified by the following fact. Let $T$ be an abstract set, and let $\widetilde{F}$ be any linear space of real-valued functions defined on $T$ such that $\max (f, 0)$ and $\min (f, 0)$ are in $\mathfrak{F}$ whenever $f \in \tilde{F}$. Furthermore, let $M$ be any real functional on the nonnegative elements of $\mathfrak{f}$ such that $M\left(f_{1}+f_{2}\right)=M\left(f_{1}\right)+M\left(f_{2}\right), M(\alpha f)=\alpha M(f)$ for $\alpha \supseteqq 0$, and $M(f) \geqslant 0$, for all non-negative $f, f_{1}$, and $f_{2} \in \tilde{F}$. Then $M$ admits a unique extension $\tilde{M}$ over $\mathscr{\wp}$ which is a linear functional: $\tilde{M}(f)=M[\max (f, 0)]$ $-M[-\min (f, 0)]$. It is an elementary exercise to verify that $\tilde{M}$ is indeed linear and unique, and we therefore omit this verification.

Two questions regarding the functional $M$ immediately present themselves: (A) Can $M$ be written as an integral? That is, does there exist a measure $\mu^{*}$ defined for some family of subsets of $X$ such that all $c \varepsilon\left(\xi_{+}\right.$are $\mu^{*}$-measurable and $M(c)=\int_{X} c(x) d \mu^{*}(x)$ for all $c \varepsilon\left(\xi_{+}\right.$? (B) Is there a natural way of extending the functional $M$ to a space of functions on $X$ which properly contains $\mathbb{E}_{+}$?

An affirmative answer to (A) is provided by the following construction, which proceeds along classical lines. For every compact subset $A$ of $X$, let $\mu(A)$ be defined as $\inf _{c \geqq \iota_{A}} M(c)$. For every open subset $G$ of $X$, let $\mu^{\prime}(G)$ be defined as $\sup _{A \varepsilon a, A \in G} \mu(A)$. For every subset $P$ of $X$, let $\mu^{*}(P)$ be defined as $\inf _{G \text { ol } \in \text { in, } G \supset P} \mu^{\prime}(G)$. The set-function $\mu^{*}$ is an outer measure on $X$ with respect to which every set in $\overline{\mathcal{F}}$ of finite $\mu^{*}$-measure is measurable. Furthermore, for $c \varepsilon \varepsilon_{+}, M(c)=\int_{X} c(x) d \mu^{*}(x)$. This outer measure $\mu^{*}$ is an extension of the measure defined by Halmos [5, pp. 216-249]. The theory of this measure has been set forth in another communication by one of the present writers [8].

Since the class of functions measurable $\left(\mu^{*}\right)$ and having finite integrals always contains functions not in $\mathbb{E}_{+}$, our affirmative answer to (A) automatically provides an affirmative answer to (B).

An extension of $M$ by a quite different construction has been described, without proofs, by H. Cartan [4, pp. 73-74]. Let $\mathfrak{H}$ denote the set of all real-valued non-negative upper semi-continuous functions $f$ on $X$ for each of which there exists a compact subset $A$ of $X$ such that $f(x)=0$ for $x \varepsilon A^{\prime}$. Let $\&$ denote the set of all lower-semi-continuous functions $g$ on $X$ such that $0 \leqq g(x) \leqq+\infty$ for all $x \in X$. For $f \in \mathfrak{l}$, let $M_{\mathfrak{l l}}(f)$ be defined as $\inf _{c \in(+, c \geqq f} M(c)$. For $g \in \mathbb{Q}$, let 
$M_{\mathcal{L}}(g)$ be defined as $\sup _{c \in C+, \subseteq \leqq g} M(c)$. For an arbitrary function $\varphi$ defined on $X$ such that $0 \leqq \varphi(x) \leqq+\infty$ for all $x \in X$, let $\bar{M}(\varphi)=\inf _{y \in \mathcal{Q}, y \geqq \varphi} M_{\mathcal{L}}(g)$, and let $\underline{M}(\varphi)$ $=\sup _{f \in \mathfrak{l}, f \leqq \varphi} M_{\mathfrak{u}}(f)$. If $\underline{M}(\varphi)=\bar{M}(\varphi)<+\infty$, then the function $\varphi$ is said to be summable and the common value of $\underline{M}(\varphi)$ and $\bar{M}(\varphi)$ is denoted by the symbol $\underline{M}(\varphi)$. The class of all summable functions is denoted by the symbol $\subseteq$.

The object of the present paper is to show that a function $\varphi$ on $X$ such that $0 \leqq \varphi(x) \leqq+\infty$ is summable if and only if it is $\mu^{*}$-measurable and has a finite integral $\int_{X} \varphi(x) d \mu^{*}(x)$; and that under these circumstances, $\int_{X} \varphi(x) d \mu^{*}(x)$ $=\underline{\bar{M}}(\varphi)$. In proving these facts, we find it necessary to establish a number of properties of the functionals $M_{\mathfrak{u}}, M_{\mathfrak{L}}, \underline{M}, \bar{M}$, and $\underline{\bar{M}}$. Accordingly, §§ 3-4 are devoted to these functionals.

§3. Properties of semi-continuous functions. For definitions and elementary properties of semi-continuous functions, we refer the reader to [2, pp. 109 -116]. We require the following facts concerning the classes $\mathfrak{U}$ and $\mathfrak{Q}$ of functions defined in $\S 2$.

3. 1 TheOREM. Let $g_{1}$ and $g_{2}$ be functions in $\mathfrak{R}$. Then $\min \left(g_{1}, g_{2}\right)$ is also a function in $L$.

3. 2 Theorem. If $f_{1}$ and $f_{2}$ are functions in $\mathfrak{H}$, then $\max \left(f_{1}, f_{2}\right) \in \mathfrak{H}$.

3. 3 THEOREM. The classes $\mathfrak{U}$ and $\mathfrak{Z}$ are closed under the operation of addition and multiplication by non-negative real numbers.

Theorems 3. 1-3.3 are simple, and the proofs are accordingly omitted.

3. 4 Theorem. Let $f \in \| l$ and $g \in \mathbb{R}$, and suppose that $f \leqq g$. Then, if $F$ is any closed set disjoint from $(E[x ; f(x)>0])^{-}$, there exists a function $\varphi \in \mathbb{C}_{+}$ such that $f \leqq \varphi \leqq g$ and $\varphi(x)=0$ for $x \in F$.

We consider first the case in which $X$ is a compact space. (This case is mentioned by N. Bourbaki [3, p. 72, exercise 27], but since the proof requires a non-obvious construction, we prefer to set it forth in extenso.) Thus we have arbitrary upper, lower respectively, semi-continuous functions $f$ and $g$ on the compact Hausdorff space $X$ such that $0 \leqq f \leqq g$. If $f(x)<g(x)$ for all $x \in X$, we easily produce a continuous function $c$ such that $f(x)<c(x)<g(x)$ for all $x \in X$. For every $x \in X$, there exists a neighborhood $U(x)$ such that $g(y)>(2 g(x)$ $+f(x)) / 3$ and $f(y)<(2 f(x)+g(x)) / 3$ for all $y \in U(x)$. Let $V(x)$ be a neighborhood of $x$ such that $V^{-}(x) \subset U(x)$ (such a $V(x)$ exists by virtue of the regularity of $X)$; and let $w_{x}$ be a continuous real function on $X$ such that 


$$
w_{x}(y)=\left\{\begin{array}{ll}
\frac{1}{2}(f(x)+g(x)) & y \in V^{-}(x) \\
0 & y \in U^{\prime}(x)
\end{array},\right.
$$

and $0<w_{x}(y) \leqq \frac{1}{2}(f(x)+g(x))$ for all $y \in X$. Since $X$ is compact, a finite number of the neighborhoods $V(x)$, say $V\left(x_{1}\right), \ldots, V\left(x_{m}\right)$, cover $X$. The function $c=\max \left(w_{x_{1}}, \ldots, w_{x_{m}}\right)$ then obviously satisfies the condition imposed. Next, consider the case where $0 \leqq f \leqq g$, but $f(x)=g(x)$ is possible. We define three sequences of functions by induction. Let $f_{1}=f, g_{1}=g$, and let $c_{1}$ be a continuous function such that $f_{1} \leqq c_{1} \leqq g_{1}+2^{-1}$. (Such a $c_{1}$ exists in view of the preceding construction.) Now suppose that the functions $f_{1}, f_{2}, \ldots, f_{n-1}, g_{1}, \ldots, g_{n-1}$, and $c_{1}, \ldots, c_{n-1}$ have been defined. We then define $f_{n}$ as $\max \left(f_{1}, c_{n-1}-2^{-(n-1)}\right)$, $g_{n}$ as $\min \left(g_{1}, c_{n-1}\right)$, and $c_{n}$ as any function in $\boldsymbol{F}_{+}$such that $f_{n} \leqq c_{n} \leqq g_{n}+2^{-n}$. Under this inductive definition, it is plain that $f_{n} \leqq g_{n}, f_{n}$ is upper semi-continuous and $g_{n}$ is lower semi-continuous; hence a function $c_{n}$ of the type required exists. It is also clear that $c_{n}-2^{-n} \leqq f_{n+1} \leqq c_{n+1} \leqq g_{n+1}+2^{-(n+1)} \leqq c_{n}+2^{-(n+1)}<c_{n}$ $+2^{-n}$. From these inequalities, upon adding, follow the inequalities

$$
\left|c_{n+p}-c_{n}\right| \leqq \sum_{k=n}^{n+p-1} 2^{-k}<2^{-n+1}
$$

The functions $c_{n}$ therefore converge uniformly, and have a continuous limit, which we denote by $c$. The inequalities

$$
f_{1} \leqq f_{n} \leqq c_{n} \leqq g_{n}+2^{-n} \leqq g_{1}+2^{-n},
$$

which are obviously valid, now show that $f \leqq c \leqq g$.

We now turn to the general case where $X$ is locally compact, $f \in \mathfrak{H}, g \in \mathbb{Q}$, and $f \leqq g$. From the construction given above, we know that on the compact set $A=(E[x, f(x)>0])$ - there exists a continuous real-valued function $\varphi_{0}$ such that $f(x) \leqq \varphi_{0}(x) \leqq g(x)$ for all $x \in A$. By an elementary construction, there exists an open set $G$ such that $A \subset G \subset G^{-} \subset F^{\prime}$ and $G^{-}$is compact. It will then suffice to show that there exists an extension $\varphi$ of $\varphi_{0}$ over $X$ such that $\varphi$ is continuous, $\varphi \geqslant 0$, and $\varphi(x)=0$ for $x \in G^{\prime}$. Consider the set 3 of all continuous real functions on $X$ which are 0 on $G^{\prime}$. Since $X$ is completely regular, it is clear that, given $p_{1}$ and $p_{2}$ distinct points of $A$, there exists a function $g \in 3$ such that $g\left(p_{1}\right)=1, g\left(p_{2}\right)=0$. Furthermore, sums, products, scalar multiples, and uniform limits of functions in 3 are again functions in 3 . Finally, since $A$ is compact, there exists a function $e \in Z$ such that $e(x)=1$ for $x \in A$. The StoneWeierstrass theorem for compact Hausdorff spaces [7] now implies that every continuous real function on $A$ coincides (on $A$ ) with some function in $\mathfrak{Z}$. Therefore $\varphi_{0}$, which is continuous on $A$, can be extended over $X$ as an element $\varphi_{1}$ of 3 ; and the function $\max \left(\varphi_{1}, 0\right)=\varphi$ satisfies the condition of the present theorem. 
3. 5 NotE. The insertion of a continuous function between two semi-continuous functions as in theorem 3.4 is not always possible in general completely regular spaces. For example, let $T$ be the space of all ordinal numbers $(\alpha, \beta)$ where $1 \leqq \alpha \leqq \Omega$ and $1 \leqq \beta \leqq \omega$, barring the point $(\Omega, \omega)$. We define $U_{\alpha, \beta}\left(\alpha_{0}, \beta_{0}\right)$ as $E\left[\left(\alpha^{\prime}, \beta^{\prime}\right) ; \alpha<\alpha^{\prime} \leqq \alpha_{0}, \beta<\beta^{\prime} \leqq \beta_{0}\right]$ for all $\alpha<\alpha_{0}$ and $\beta<\beta_{0}$. These sets $U_{*, \beta}\left(\alpha_{0}, \beta_{0}\right)$ are then a complete system of neighborhoods of $\left(\alpha_{0}, \beta_{0}\right)$. Let $G=E[(\alpha, n)$; $1 \leqq \alpha \leqq \Omega, n=1,3,5, \ldots]$. Let $F=E[(\Omega, n) ; n=1,3,5, \ldots]$. Then $\ell=$ is lower semi-continuous, $\iota_{F}$ is upper semi-continuous, and $\iota_{F} \leqq \iota_{i}$. However, it is not hard to prove that any real-valued continuous function $\varphi$ such that $\varphi \oslash_{F}$ must necessarily have values $\geqq 1$ on a set of the form $E\left[\left(\alpha_{0}, \beta\right) ; n \leqq \beta \leqq \omega\right]$ for some $\alpha_{0}<\Omega$ and $n<\omega$. Hence the relations $\iota_{F} \leqq \varphi \leqq \iota_{G}$ cannot be simultaneously satisfied. The space $T$, of course, is non-normal, and this fact is utilized heavily in the above construction. The question, so far as the writers are aware, remains open for non-compact normal spaces.

$\S$ 4. Properties of the functionals $M_{\mathfrak{l}}, M_{\mathcal{L}}, M, \bar{M}$, and $\bar{M}$.

4. 1 Theorem. Let $f \in \mathfrak{U}$ and let $\alpha$ be any non-negative real number: Then $M_{\mathfrak{U}}(\alpha f)=\alpha M_{\mathfrak{n}}(f)$.

If $\alpha=0$, this equality is obvious. For $\alpha>0$, and $\varepsilon>0$, let $c$ be a function in $\varepsilon_{+}$such that $c \supseteqq f$ and $M(c)-\frac{\varepsilon}{\alpha}<M_{\mathfrak{u}}(f)$. Then $\alpha c \supseteqq \alpha f$, and thus $\alpha M(c)$ $=M(\alpha c) \supseteqq M_{\mathfrak{U}}(\alpha f)$; on the other hand, $\alpha M(c)<\alpha M_{\mathfrak{U}}(f)+\varepsilon$. Thus $M_{\mathfrak{l}}(\alpha f)$ $\left\langle\alpha M_{\mathfrak{u}}(f)+\varepsilon\right.$ for all $\varepsilon>0$, and hence $M_{\mathfrak{U}}(\alpha f) \leqq \alpha M_{\mathfrak{l}}(f)$. Replacing $f$ by $\frac{1}{\alpha} f$ and multiplying through by $\frac{1}{\alpha}$, we have $\frac{1}{\alpha} M_{\mathfrak{l}}(f) \leqq M_{\mathfrak{l n}}\left(\frac{1}{\alpha} f\right)$; upon replacing $\frac{1}{\alpha}$ by $\alpha$, we have $\alpha M_{\mathfrak{l}}(f) \leqq M_{\mathfrak{u}}(\alpha f)$; and this establishes 4. 1 .

4. 2 Theorem. Let $g \in \mathbb{Q}$ and let $\alpha$ be any non-negative real number. Then $M_{\mathcal{Q}}(\alpha g)=\alpha M_{\mathfrak{Q}}(g)$.

The proof of 4.2 is very like that of 4.1 .

4. 3 TheOREM. Let $f_{1}$ and $f_{2}$ be any functions in $\mathfrak{u}$. Then $M_{\mathfrak{n}}\left(f_{1}+f_{2}\right)$ $=M_{\mathfrak{u}}\left(f_{1}\right)+M_{\mathfrak{u}}\left(f_{\mathfrak{g}}\right)$.

Let $\varepsilon$ be any positive real number, and let the functions $c_{i} \in \mathbb{S}_{+}$have the property that $c_{1} \geqq f_{i}$ and $M\left(c_{i}\right)-\frac{\varepsilon}{2}<M_{\mathfrak{U}}\left(f_{i}\right)(i=1,2)$. Then clearly $M_{\mathfrak{l}}\left(f_{1}+f_{\mathfrak{g}}\right)$ $\leqq M\left(c_{1}+c_{2}\right)=M\left(c_{1}\right)+M\left(c_{2}\right)<M_{\mathfrak{l}}\left(f_{\mathfrak{3}}\right)+M_{\mathfrak{l}}\left(f_{2}\right)+\varepsilon$. This proves that $M_{\mathfrak{l}}\left(f_{1}+f_{2}\right)$ $\leqq M_{\mathfrak{u}}\left(f_{1}\right)+M_{\mathfrak{u}}\left(f_{2}\right)$. Conversely, suppose that $c \in \mathbb{S}_{+}$, that $c \geqq f_{1}+f_{2}$, and that $M(c)-\varepsilon<M_{\mathfrak{n}}\left(f_{1}+f_{2}\right)$. It is clear that $c-f_{2} \in \mathcal{Q}$ and that $f_{1} \leqq c-f_{2}$. By 3.4, there exists a function $c_{1} \in\left(5_{+}\right.$such fhat $f_{1} \leqq c_{1} \leqq c-f_{2}$. It is clear that $c-c_{1} \in\left(5_{+}\right.$and 
that $f_{2} \leqq c-c_{1}$. Writing $c-c_{1}$ as $c_{2}$, we then have $c_{1}+c_{2}=c$, and $c_{1} \triangleq f_{1}, c_{2} \triangleq f_{2}$. The following relations are now evident: $M_{\mathfrak{n}}\left(f_{1}\right)+M_{\mathfrak{u}}\left(f_{2}\right) \leqq M\left(c_{1}\right)+M\left(c_{2}\right)$ $=M\left(c_{1}+c_{2}\right)=M(c)<M_{\mathfrak{u}}\left(f_{1}+f_{2}\right)+\varepsilon$. From this, we infer that $M_{\mathfrak{u}}\left(f_{\mathfrak{1}}\right)+M_{\mathfrak{u}}\left(f_{2}\right)$ $\leqq M_{\mathfrak{U}}\left(f_{1}+f_{2}\right)$ and thus establish the present theorem.

4. 4 Theorem. Let $g_{1}$ and $g_{2}$ be any functions in 2 . Then $M_{\mathfrak{Q}}\left(g_{1}+g_{2}\right)$ $=M_{\mathcal{L}}\left(g_{1}\right)+M_{\mathcal{L}}\left(g_{2}\right)$.

If $M_{\mathfrak{Q}}\left(g_{1}\right)$ or $M_{\mathfrak{Q}}\left(g_{2}\right)$ is $+\infty$, it is obvious that $M_{\mathfrak{Q}}\left(g_{1}+g_{2}\right)=+\infty=M_{\mathfrak{Q}}\left(g_{1}\right)$ $+M_{\Omega}\left(g_{2}\right)$. We therefore assume that both $M_{\mathcal{Q}}\left(g_{1}\right)$ and $M_{\mathcal{Q}}\left(g_{2}\right)$ are finite. Let $c_{i} \in \mathbb{S}_{+}$have the properties that $c_{i} \leqq g_{i}$ and $M\left(c_{i}\right)+\frac{\varepsilon}{2}>M_{\mathcal{\Omega}}\left(g_{i}\right) \quad(i=1,2)$. We then have $M_{\mathfrak{R}}\left(g_{1}+g_{2}\right) \supseteqq M\left(c_{1}+c_{2}\right)=M\left(c_{1}\right)+M\left(c_{2}\right)>M_{\mathfrak{L}}\left(g_{1}\right)+M_{\mathcal{R}}\left(g_{2}\right)-\varepsilon$, and accordingly, $M_{\mathcal{Q}}\left(g_{1}+g_{2}\right) \equiv M_{\mathcal{L}}\left(g_{1}\right)+M_{\mathcal{Q}}\left(g_{2}\right)$. In proving the converse inequality, we consider first the case in which $M_{\Omega}\left(g_{1}+g_{2}\right)<+\infty$. Let $c \in \mathbb{S}_{+}$be such that $c \leqq g_{1}+g_{2}$ and $M(c)+\varepsilon>M_{\mathcal{2}}\left(g_{1}+g_{2}\right)$. Then $\max \left(c-g_{1}, 0\right)$ is an upper semicontinuous function vanishing outside the compact set $(E[x, c(x)>0])^{-}$. Accordingly, there exists a function $c_{2} \in \mathbb{S}_{+}$such that $\max \left(c-g_{1}, 0\right) \leqq c_{2} \leqq g_{2}$. Now the function $c-c_{2}$ has the property that $c-c_{2} \leqq g_{1}$, as a simple calculation shows. Hence, writing $\max \left(c-c_{2}, 0\right)$ as $c_{1}$, we have $c_{1} \leqq g_{1}, c \leqq c_{1}+c_{2}$ and therefore $M_{\mathfrak{R}}\left(g_{1}\right.$ $\left.+g_{2}\right)-\varepsilon<M(c) \leqq M\left(c_{1}+c_{2}\right)=M\left(c_{1}\right)+M\left(c_{2}\right) \leqq M_{\mathfrak{Q}}\left(g_{1}\right)+M_{\mathcal{L}}\left(g_{2}\right)$. The case $M_{\mathfrak{Q}}\left(g_{1}+g_{2}\right)$ $=+\infty$ is handled similarly, using, for an arbitrarily large positive real number $A$, a function $c \in \mathcal{C}_{+}$such that $c \leqq g_{1}+g_{2}$ and $M(c)>A$. The inequality $M_{\Omega}\left(g_{1}+g_{2}\right)$ $\leqq M_{\mathcal{Q}}\left(g_{1}\right)+M_{\mathcal{Q}}\left(g_{2}\right)$ is thus established in all cases, and with it the present theorem.

4.5 Theorem. Every function $c$ of $\left(s_{+}\right.$is in $\mathfrak{l}$ and in $\mathfrak{L}$ and $M(c)=M_{\mathfrak{u}}(c)$ $=M_{\mathbb{Q}}(c)$.

4. 6 THEOREM. If $\psi_{1}$ and $\psi_{2}$ are in $\mathfrak{U}$ (or $\left.\mathfrak{Q}\right)$ and if $\psi_{1} \leqq \psi_{2}$, then $M_{\mathfrak{u}}\left(\psi_{1}\right) \leqq M_{\mathfrak{n}}\left(\psi_{2}\right)$ (or $\left.M_{\mathcal{Q}}\left(\psi_{1}\right) \leqq M_{\mathcal{L}}\left(\psi_{2}\right)\right)$.

4. 7 THEOREM. If $\psi_{1}, \psi_{2}$, and $\psi_{1}-\psi_{2}$ are in $\mathfrak{u}($ or $\mathfrak{Q})$, then $M_{\mathfrak{l}}\left(\psi_{1}-\psi_{2}\right)=M_{\mathfrak{u}}\left(\psi_{1}\right)$ $-M_{\mathfrak{l}}\left(\psi_{2}\right)\left(\right.$ or $\left.M_{\mathfrak{L}}\left(\psi_{1}-\psi_{2}\right)=M_{\mathfrak{L}}\left(\varphi_{1}\right)-M_{\mathfrak{L}}\left(\psi_{2}\right)\right)$.

Theorems 4.5-4.7 are obvious.

We now consider the functionals $\underline{M}, \bar{M}$, and $\underline{M}$ defined in $\S 2$ (q.v.).

4.8 TheOREM. For every function $\varphi$ on $X$ such that $0 \leqq \varphi(x) \leqq+\infty, \underline{M}(\varphi)$ $\leqq \bar{M}(\varphi)$.

Let $f$ and $g$ be functions in $U$ and $L$ respectively such that $f \leqq \varphi \leqq g$. Then, by 3.4 , there is a function $c \in\left(\xi_{+}\right.$such that $f \leqq c \leqq g$. Thus $M_{\mathfrak{l l}}(f) \leqq M(c) \leqq M_{\mathfrak{L}}(g)$, and it follows that $M(\varphi)=\sup _{f \in \mathfrak{U}, f \leqq p} M_{\mathfrak{l l}}(f) \leqq \inf _{\mathfrak{g} \in \mathcal{Q}, g \geq ?} M_{\mathfrak{Q}}(g)$. 
4. 8a Theorem. Let $g \in \mathfrak{Q}$. Then $M_{\mathfrak{L}}(g) \leqq \underline{M}(g)$, and $\bar{M}(f) \leq M_{\mathfrak{u}}(f)$ for $f \in U$.

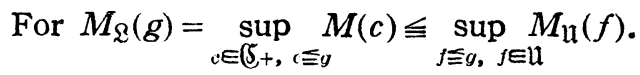

4.9 Theorem. Let $\varphi_{1}$ and $\varphi_{2}$ be functions in $\Im$. Then $\varphi_{1}+\varphi_{2} \in \Im$.

Let $\varepsilon$ be any positive real number. Then there exist functions $f_{i} \in \mathfrak{U}$ and $g_{i} \in \mathcal{L}$ such that $f_{i} \leqq \varphi_{i} \leqq g_{i}$ and $M_{\mathfrak{u}}\left(f_{i}\right)+\frac{\varepsilon}{2}>M_{\mathfrak{L}}\left(g_{i}\right) \quad(i=1,2)$. Then $f_{1}+f_{2} \leqq \varphi_{1}+\varphi_{2}$ $\leqq g_{1}+g_{2}$, and $M_{\mathfrak{U}}\left(f_{1}+f_{2}\right)=M_{\mathfrak{l}}\left(f_{1}\right)+M_{\mathfrak{U}}\left(f_{2}\right)>M_{\mathfrak{L}}\left(g_{1}\right)+M_{\mathfrak{L}}\left(g_{2}\right)-\varepsilon=M_{\mathfrak{L}}\left(g_{1}+g_{2}\right)-\varepsilon$. Hence $\underline{M}\left(\varphi_{1}+\varphi_{2}\right) \supseteqq \bar{M}\left(\varphi_{1}+\varphi_{2}\right)$, and by 4.8 , equality must obtain. It is also clear that $\underline{M}\left(\varphi_{1}+\varphi_{2}\right)$ is finite.

4.10 THEOREM. If $\varphi \in \mathbb{S}$ and $\alpha$ is a non-negative real number, then $\alpha \varphi \in \mathbb{S}$.

4. 11 THEOREM. If $\varphi_{1}$ and $\varphi_{2} \in S$, then $\underline{\bar{M}}\left(\varphi_{1}+\varphi_{2}\right)=\underline{\bar{M}}\left(\varphi_{1}\right)+\underline{\bar{M}}\left(\varphi_{2}\right)$. If $\varphi \in \mathbb{S}$ and $\alpha$ is a non-negative real number, then $\underline{\bar{M}}(\alpha \varphi)=\alpha \bar{M}(\varphi)$. If $\varphi_{1}, \varphi_{2} \in \subseteq$ and $\varphi_{1} \supseteqq \varphi_{2}$, then $\varphi_{1}-\varphi_{2} \in \Im$, and $\underline{\bar{M}}\left(\varphi_{1}-\varphi_{2}\right)=\underline{\bar{M}}\left(\varphi_{1}\right)-\overline{\bar{M}}\left(\varphi_{2}\right)$.

4.10 and 4.11 are established in the usual way.

4.12 Theorem. Let $g$ be any function of $\mathcal{L}$. Then $g \in \Im$ if and only if. $M_{\mathfrak{Q}}(g)$ is finite; and in this case $\underline{\bar{M}}(g)=M_{\mathfrak{L}}(g)$.

Plainly $g \in \Im$ implies that $M_{\mathfrak{\Omega}}(g)$ is finite ; for $M_{\mathcal{L}}(g) \leqq \underline{M}(g)$ by $4.8 \mathrm{a}$. Conversely, if $M_{\Omega}(g)$ is finite, then, by $4.6, \bar{M}(g) \leqq M_{\Omega}(g)$. Since $M_{\mathcal{L}}(g) \leqq \underline{M}(g)$, we have $\bar{M}(g) \leqq \underline{M}(g)$, and thus, in view of $4.8, \underline{M}(g)=\bar{M}(g)=M_{\mathfrak{L}}(g)=\underline{\bar{M}}(g)$; and plainly $\underline{\bar{M}}(g)<+\infty$.

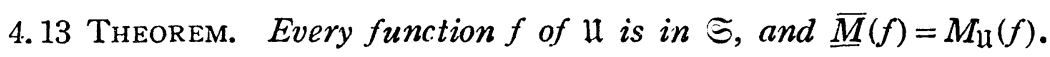

It follows from 4.6 that $\underline{M}(f)=M_{\mathfrak{u}}(f)$, and from $4.8 \mathrm{a}$ that $M(f) \leqq M_{\mathfrak{l l}}(f)$. This, with 4.8 , completes the present proof.

4. 14 THEOREM. Let $\psi$ be a non-negative upper semi-continuous function on $X$ such that $\psi \leqq g$, where $g \in \mathbb{R}$ and $M_{\mathfrak{Q}}(g)<+\infty$. Then $\psi$ is summable.

Since $\psi \leqq g$ we have $\underline{M}(\psi) \leqq M(g)=M_{\mathfrak{Q}}(g)<+\infty$, and we need only to prove that $\bar{M}(\psi)=\underline{M}(\psi)$. It is clear that $\underline{M}(\psi)=\sup _{A \in a} M_{\mathfrak{U}}\left(\psi_{A}\right)$, since every $M_{\mathfrak{l l}}\left(\psi_{A}\right)$ is $\leqq \underline{M}(\psi)$ and every $f \in \mathfrak{U}$ such that $f \leqq \psi$ is majorized by some $\psi_{A}$. Let $\varepsilon$ be any positive number. Since $M_{\mathfrak{L}}(g)$ is finite, there exists a function $d \in \mathfrak{E}_{+}$, vanishing outside of a compact set $D$, such that $d \leqq g$ and $M(d)+\varepsilon>\mathrm{M}_{\mathcal{L}}(g)$. We next observe that $g_{D^{\prime}}$ is in $\mathbb{Q}$, that $g_{D^{\prime}} \leqq g-d$, that $M_{\mathcal{L}}(g-d)=M_{\mathfrak{L}}(g)-M(d)$, and that accordingly $M_{\Omega^{\prime}}\left(g_{D^{\prime}}\right)<\varepsilon$. By 3.4, there exists a function $c_{1} \in\left(_{+}\right.$such that $\psi_{D} \leqq c_{1} \leqq g$. By the definition of $M_{\mathfrak{l}}\left(\psi_{D}\right)$, there exists a function $c_{\mathfrak{g}} \in \overleftarrow{E}_{+}$such that $c_{2} \triangleq \psi_{D}$ and $M\left(c_{2}\right)<M_{\mathfrak{U}}\left(\psi_{D}\right)+\varepsilon$. Denoting $\min \left(c_{1}, c_{2}\right)$ by $c$, we obviously have $c \in \mathcal{F}_{+}, g \equiv c \supseteqq \psi_{D}$, and $M(c)<M_{\mathfrak{l}}\left(\psi_{D}\right)+\varepsilon$. The function $c+g_{D^{\prime}}$ is obviously in $\mathfrak{Z}$ and satisfies the 
inequality $\psi \leqq c+g_{D^{\prime}}$. We apply the definitions of $\bar{M}$ and $\underline{M}, 4.4$, and the inequalities developed above to write the following: $\bar{M}(\psi) \leqq M_{\mathbb{R}}\left(c+g_{D^{\prime}}\right)=M_{\mathfrak{L}}(c)$ $+M_{\mathfrak{L}}\left(g_{D^{\prime}}\right)=M(c)+M_{\mathfrak{L}}\left(g_{D^{\prime}}\right)<M(c)+\varepsilon<M_{\mathfrak{U}}\left(\psi_{D}\right)+2 \varepsilon \leqq \underline{M}(\psi)+2 \varepsilon$. Accordingly, $\bar{M}(\psi) \leqq \underline{M}(\psi)$, and by $4.8, \bar{M}(\psi)=\underline{M}(\psi)$.

4.15 THEOREM. Let $\left\{g_{n}\right\}_{n=1}^{\infty}$, be a countably infinite sequence of functions of \& such that $g_{1} \leqq g_{2} \leqq g_{3} \leqq \ldots \leqq g_{n} \leqq \ldots$ Then $M_{\mathfrak{L}}\left(\sup _{1 \leqq n<\infty} g_{n}\right)=\sup _{1 \leqq n<\infty} M_{\mathfrak{L}}\left(g_{n}\right)$.

Write $\sup _{1 \leqq n<\infty} g_{n}=g$. Then we have, obviously, $g_{n} \leqq g$ and $M_{\mathfrak{L}}\left(g_{n}\right) \leqq M_{\mathfrak{L}}(g)$ for all $n$. We must therefore show that $\lim _{n \rightarrow \infty} M_{\mathfrak{L}}\left(g_{n}\right) \geqq M_{\mathfrak{L}}(g)$. Consider first the case $M_{\mathfrak{I}}(g)<+\infty$. Let $\varepsilon_{1}$ and $\varepsilon_{2}$ be any positive numbers, and let $c$ be a function of $\left(\xi_{+}\right.$such that $c \leqq g$ and $M(c)+\frac{\varepsilon_{1}}{2}>M_{\mathfrak{L}}(g)$. Let $A=(E[x, c(x)>0])^{-}$; clearly $A$ is compact. For every $a \in A$, there exists an integer $n(a)$ such that $g_{n(a)}(a)$ $>g(a)-\frac{\varepsilon_{2}}{4}$ and a neighborhood $U(a)$ such that $|c(x)-c(a)|<\frac{\varepsilon_{2}}{2}$ for $x \in U(a)$ and $g_{n(a)}(x)>g_{n(a)}-\frac{\varepsilon_{2}}{4}$ for $x \in U(a)$. Then clearly $g_{n(a)}(x)>c(x)-\varepsilon_{2}$ for all $x \in U(a) . \quad A$ finite number of these neighborhoods, say $U\left(a_{1}\right), \ldots, U\left(a_{k}\right)$, cover A. Writing $n_{0}=\max \left(n\left(a_{1}\right), \ldots, n\left(a_{k}\right)\right)$, we have $g_{n_{0}}(x)>c(x)-\varepsilon_{2}$ for all $x \in A$. Next, let $c_{0}$ be any function in $\xi_{+}$which is $\geqq 1$ on $A$. Since $c=0$ on $A^{\prime}$, the following inequalities hold: $c-\min \left(g_{n_{0}}, c\right) \leqq \varepsilon_{2} c_{0}, c \leqq \varepsilon_{2} c_{0}+\min \left(g_{n_{0}}, c\right)$. Then the monotonicity and additivity of $M_{\mathfrak{L}}$ show that $M(c)=M_{\mathfrak{L}}(c) \leqq \varepsilon_{2} M\left(c_{0}\right)+M_{\mathfrak{L} \min }\left(g_{n_{0}}\right.$, $c) \leqq \varepsilon_{2} M\left(c_{0}\right)+M_{\mathcal{Q}}\left(g_{n_{0}}\right)$. Choosing $\varepsilon_{2}<\frac{\varepsilon_{1}}{2 M\left(c_{0}\right)}$ (as we are obviously at liberty to do), we have $M(c)<\frac{\varepsilon_{1}}{2}+M_{\mathfrak{L}}\left(g_{n_{0}}\right)$. Thus we have $M_{\mathfrak{L}}(g)-\frac{\varepsilon_{1}}{2}<M(c)<\frac{\varepsilon_{1}}{2}+M_{\mathfrak{L}}\left(g_{n_{0}}\right)$, and consequently $M_{\mathfrak{L}}(g) \leqq \lim _{n \rightarrow \infty} M_{\mathfrak{L}}\left(g_{n}\right)$. If $M_{\mathfrak{L}}(g)=+\infty$, we choose $c \in \mathbb{S}_{+}$such that $c \leqq g$ and $M(c)$ is arbitrarily large. The argument presented above is then repeated.

§. The measure $\mu^{*}$.

5.1 TheOREM. Let $A$ be any compact subset of $X$. Then $\iota_{A} \in \mathfrak{l}$ and $\bar{M}\left(\iota_{A}\right)$ $=\mu(A)$.

These observations are obvious from the definitions of $\mathfrak{u}, \mu$, and $M_{\mathfrak{u}}$, and from 4.13.

5. 2 Theorem. Let $G$ be any open subset of $X$. Then $\ell_{G} \in \mathbb{Q}$ and $\mu^{\prime}(G)=M_{\mathcal{L}}\left(\varsigma_{i}\right)$. Thus ${ }_{Q} G$ is summable if and only if $\mu^{\prime}(G)$ is finite.

It is obvious that $\ell_{G} \in$ ?. As is stated in $\S 2$, we have $\mu^{\prime}(G)=\sup _{A \in a, A \in C} \mu(A)$. In proving that $M_{\Omega}\left(\iota_{G}\right) \supseteqq \mu^{\prime}(G)$, suppose first that $\mu^{\prime}(G)$ is finite. Then, for every $\varepsilon>0$, there is a set $A \in a$ such that $A \subset G$ and $\mu(A)+\varepsilon>\mu^{\prime}(G)$. By 3.4, setting 
$f=\ell_{A}$ and $g=\ell_{G}$, there exists a function $c \in \mathscr{E}_{+}$such that

$$
c(x)=\left\{\begin{array}{ll}
1 & x \in A \\
0 & x \in G^{\prime}
\end{array} \text { and } 0 \leqq c(x) \leqq 1 \text { for all } x .\right.
$$

The following inequalities are now clear: $M_{\mathcal{L}}\left(\iota_{G}\right)+\varepsilon \supseteqq M(c)+\varepsilon \equiv \mu(A)+\varepsilon>\mu^{\prime}(G)$. If $\mu^{\prime}(G)$ is infinite, we select an $A \in a$ and contained in $G$ such that $\mu(A)$ is arbitrarily large and repeat the argument. Thus $M_{\Omega}\left(\iota_{G}\right) \geqq \mu^{\prime}(G)$ in any case. To prove that $M_{\mathcal{L}}\left(\ell_{G}\right) \leqq \mu^{\prime}(G)$, we consider first the case $M_{\mathcal{L}}\left(\varrho_{G}\right)<+\infty$. Then, for an arbitrary $\varepsilon>0$, we select a function $c \in \varsigma_{+}$such that $c \leqq ! G$ and $M(c)+\frac{\varepsilon}{2}$ $>M_{\mathfrak{Q}}\left(\ell_{\mathfrak{G}}\right)$. Next, let $\alpha$ be a real number such that $0<\alpha<1$. The set $B_{\alpha}=E[x$; $c(x) \supseteqq \alpha]$ is a compact subset of $G$. Then, by 3.4 , we may choose a function $d \in \complement_{+}$such that

$$
\begin{aligned}
d(x)= \begin{cases}1 & x \in B_{\alpha} \\
0 & x \in G^{\prime} \\
& 0 \leqq d(x) \leqq 1 \text { all } x \in X .\end{cases}
\end{aligned}
$$

Writing $\max [c(x), d(x)]-d(x)=h(x)$, we clearly have $\alpha>h(x) \geqq 0$ for all $x$ and $h(x)=0$ for $x \in G^{\prime}$. Thus $h<\alpha \ell_{G}$, and by 4.5 and 4.6 , we have $M(h) \leqq \alpha M_{\mathscr{Q}}\left(\ell_{G}\right)$. It follows that $M(c)-M(d) \leqq M(\max (c, d))-M(d)=M(h) \leqq \alpha M_{\mathfrak{L}}\left(\iota_{G}\right)$. From this we infer that

$$
M_{\mathfrak{L}}\left(\ell_{G}\right)<M(c)+\frac{\varepsilon}{2} \leq M(d)+\alpha M_{\mathfrak{L}}\left(\ell_{G}\right)+\frac{\varepsilon}{2} .
$$

Choosing $\alpha<\frac{\varepsilon}{2 M_{\mathfrak{Q}}\left(\ell_{G}\right)}$ (the trivial case $M_{\mathfrak{Q}}\left(\ell_{G}\right)=0$ can be ignored here), and taking the infimum over all $d$ satisfying the conditions imposed above, we have $M_{\mathfrak{L}}\left(\ell_{G}\right) \leqq \mu\left(B_{\alpha}\right)+\varepsilon$. Since $\mu\left(B_{\alpha}\right) \leqq \mu^{\prime}(G)$, the inequality $M_{\mathscr{Q}}\left(\ell_{G}\right) \leqq \mu^{\prime}(G)$ is established. If $M_{\mathcal{Q}}\left(\iota_{G}\right)=+\infty$, we select a function $c \in\left(_{+}\right.$such that $c \leqq \iota_{G}$ and $M(c)$ is arbitrarily large, and proceed as before. Thus the present theorem is established.

\subsection{THEOREM. Let $P$ be any subset of $X$. Then $\mu^{*}(P)=\bar{M}\left(\iota_{P}\right)$.}

We prove first that $\bar{M}\left(\iota_{P}\right) \leqq \mu^{*}(P)$. We may suppose that $\mu^{*}(P)<+\infty$, that $\varepsilon$ is a positive real number, and that $G$ is an open set $\supset P$ such that $\mu^{\prime}(G)-\varepsilon$ $<\mu^{*}(P)$. Then the function $\iota_{G}$ is in $\mathcal{L}$ and is $\triangleq \ell_{P}$; by 5.2 and the definition of $\bar{M}$, we have $\mu^{*}(P)+\varepsilon>\mu^{\prime}(G)=M_{\mathfrak{Q}}\left(\iota_{G}\right) \geqslant \bar{M}\left(\iota_{P}\right)$. Hence $\bar{M}\left(\iota_{P}\right) \leqq \mu^{*}(P)$. In proving the reverse inequality, we suppose that $\bar{M}\left(\ell_{P}\right)$ is finite, that $\varepsilon$ is an arbitrary positive number, and that $g$ is a function in $\mathfrak{Q}$ such that $g(x)=1$ for $x \in P$ and $M_{\mathfrak{Q}}(g)<\frac{\varepsilon}{2}+\bar{M}\left(\iota_{P}\right)$. Let $\alpha$ be any number such that $0<\alpha<1$. Then the set $D_{\alpha}=E[x ; g(x)>\alpha]$ is an open set containing $P$. Consider the function $\psi(x)$ 
$=\max \left[c_{\alpha}(x), g(x)\right]-g(x)$. We have

$$
\psi(x)=\left\{\begin{array}{cc}
1-g(x) & x \in D_{\alpha} \\
0 & x \text { non } \in D_{\alpha}
\end{array},\right.
$$

and the inequalities $0 \leqq \psi(x) \leqq\left(\frac{1}{\alpha}-1\right) g(x)$ are easily verified. It is also obvious that $\psi$ is upper semi-continuous. Being bounded above by the summable function $\left(\frac{1}{\alpha}-1\right) g, \phi$ is also summable (4.14), and we have $\underline{\bar{M}}(\psi+g)=\underline{\bar{M}}(\psi)+M_{\mathfrak{L}}(g)$ $=M_{\mathcal{L}}\left(\max \left(\ell_{D_{\alpha}}, g\right)\right) \geqq M_{\mathcal{L}}\left(\ell_{D_{\alpha}}\right)$. Furthermore, the inequality $\psi \leqq\left(\frac{1}{\alpha}-1\right) g$ implies that $\underline{\bar{M}}(\psi) \leqq\left(\frac{1}{\alpha}-1\right) M_{\mathfrak{R}}(g)$. Assembling the results obtained so far, we have $\mu^{*}(P) \leqq \mu^{\prime}(D)=M_{\mathcal{L}}\left(\ell_{\alpha}\right) \leqq M_{\mathcal{L}}(g)+\underline{\bar{M}}(\psi) \leqq M_{\mathcal{L}}(g)+\left(\frac{1}{\alpha}-1\right) M_{\mathcal{L}}(g)<\frac{\varepsilon}{2}+\left(\frac{1}{\alpha}-1\right) M_{\mathcal{L}}(g)$ $+\bar{M}\left(\iota_{P}\right)$. By choosing $\alpha$ sufficiently close to 1 , we make $\left(\frac{1}{\alpha}-1\right)<\frac{\varepsilon}{2 M_{\Omega}(g)}$ and thus establish the inequality $\mu^{*}(P) \leqq M\left(\iota_{P}\right)$.

5.4 Theorem. Let $T$ be any subset of $X$ such that ${ }_{i_{T}}$ is summable. Then there exists a compact subset $A$ of $T$ such that $\mu(A)+\varepsilon>\underline{\bar{M}}\left({ }_{(\mathrm{T}}\right)$, where $\varepsilon$ is an arbitrary positive real number.

Suppose first that $\underline{\bar{M}}\left(\iota_{T}\right)>0$. Let $f$ be a function in $\mathfrak{u}$ such that $f \leqq \iota_{T}$ and $M_{\mathfrak{l}}(f)+\frac{\varepsilon}{2}>\underline{\bar{M}}\left(\mathfrak{l}_{T}\right)$. For every number $\alpha$ such that $0<\alpha \leqq 1$, the set $B_{\alpha}=E[x$; $f(x) \triangleq \alpha]$ is a compact subset of $T$. It is obvious that $\iota_{B_{\alpha}}+\alpha \iota_{T} \geqq f$. As the characteristic function of a compact set, $\iota_{B \alpha}$ is in $\mathfrak{u}$ and by 4.13 is in $\Im$. Applying $5.1,4.10,4.13$, and the monotonicity of $\underline{\bar{M}}$, we find $\mu\left(B_{\alpha}\right)+\alpha \underline{\bar{M}}\left(\iota_{T}\right)=\underline{\bar{M}}\left(\iota_{B_{\alpha}}\right)$ $+\alpha \underline{\bar{M}}\left(\iota_{T}\right)=\underline{\bar{M}}\left(\iota_{B_{a}}+\alpha_{\imath_{T}}\right) \geqq \underline{\bar{M}}(f)=M_{\mathfrak{H}}(f)>\underline{\bar{M}}\left(\iota_{T}\right)-\frac{\varepsilon}{2}$. Choosing $\alpha<\frac{\varepsilon}{2 \bar{M}\left(\iota_{T}\right)}$, we have $\mu\left(B_{\alpha}\right)+\varepsilon>\bar{M}\left(\iota_{T}\right)$, as we wished to show. The case $\underline{\bar{M}}\left(\iota_{T}\right)=0$ is simple, since in this event, if $A$ is a compact subset of $T, \underline{\bar{M}}\left(\iota_{A}\right)=\mu(A) \leqq \underline{\bar{M}}\left(\iota_{T}\right)=0$ of necessity.

We next make a few remarks on $\mu^{*}$-measurability.

5.5 'Theorem. Let $G$ be any open subset of $X$ such that $\mu^{\prime}(G)=\mu^{*}(G)$ is finite. Then there exist a family $\left\{A_{n}\right\}_{n=1}^{\infty}$ of compact sets and a set $N$ of $\mu^{*}$ measure 0 such that $G=\left(\bigcup_{n=1}^{\infty} A_{n}\right) \cup N ; G$ is therefore $\mu^{*}$-measurable.

By 5.2, $\iota_{G}$ is in $\subseteq$. Then, by 5.4, there exists for every positive integer $n$ a compact subset $A_{n}$ of $G$ such that $\mu\left(A_{n}\right)+\frac{1}{n}>\underline{M}\left(\iota_{G}\right)$. Let $B_{n}=\bigcup_{j=1}^{n} A_{j}$. Plainly $\mu\left(B_{n}\right)+\frac{1}{n}>\underline{M}\left(\iota_{G}\right)$. Now the sets $B_{n}$, being compact, are $\mu^{*}$-measurable [8, Th. 3]. As the union of a countable family of $\mu^{*}$-measurable sets, the set $P=\bigcup_{n=1}^{\infty} A_{n}=\bigcup_{n=1}^{\infty} B_{n}$ is $\mu^{*}$-measurable. By a well-known theorem $\left[6\right.$, p. 8 , Th. 5.1], we have $\mu^{*}(P)$ 
$=\lim _{n \rightarrow \infty} \mu\left(B_{n}\right)$, and hence $\mu^{*}(P) \equiv M\left(\bullet_{G}\right)$. On the other hand, every $B_{n}$ is a compact subset of $G$, and $\mu\left(B_{n}\right)=\underline{\bar{M}}\left(\ell_{B_{n}}\right) \leqq \underline{M}\left(\ell_{G}\right)$, and thus $\lim _{n \rightarrow \infty} \mu\left(B_{n}\right) \leqq \underline{M}\left(\iota_{G}\right)$. Therefore $\mu^{*}(P)=\underline{M}\left(\iota_{G}\right)$. However, $\underline{M}\left(\iota_{G}\right)=\bar{M}\left(\iota_{G}\right)=\mu^{*}(G)$, by 4.12 and 5.3. Since $P$ is $\mu^{*}$-measurable, we have $\mu^{*}(G)=\mu^{*}(G \cap P)+\mu^{*}\left(G \cap P^{\prime}\right)=\mu^{*}(P)+\mu^{*}\left(G \cap P^{\prime}\right)$. Hence $\mu^{*}\left(G \cap P^{\prime}\right)=0$; writing $G \cap P^{\prime}$ as $N$, and noting that every set of $\mu^{*}$-measure 0 is $\mu^{*}$-measurable, we have the present theorem.

5. 6 THEOREM. Let $F$ be any closed subset of $X$ such that $\mu^{*}(F)$ is finite. Then $F$ is $\mu^{*}$-measurable, and can be written in the form $\bigcup_{n=1}^{\infty} C_{n} \cup L$, where the $C_{n}$ are compact and $\mu^{*}(L)=0$.

Since $\mu^{*}(F)$ is finite, there exists an open set $G \supset F$ such that $M_{2}\left(\iota_{G}\right)=\mu^{\prime}(G)$ $<\infty$. The function $l_{F}$, which is certainly upper semi-continuous, is therefore summable, by 4.14. The argument now follows that used in 5.5.

5.6a Corollary. Let $Z$ be any subset of $X$ such that $Z=\bigcup_{n=1}^{\infty} Z_{n}$, where $Z_{n} \in \overline{\mathcal{F}}$ and $\mu^{*}\left(Z_{n}\right)<\infty$. Then $Z$ is $\mu^{*}$-measurable.

This is an immediate consequence of the fact that each $Z_{n}$ is contained in an open set $G_{n}$ of finite $\mu^{*}$-measure which is $\mu^{*}$-measurable by 5.5 , and that the family of $\mu^{*}$-measurable sets is closed under the formation of countable unions and of complements.

5.7 Theorem. Let $T$ be a $\mu^{*}$-measurable subset of $X$ such that $\mu^{*}(T)<\infty$. Then there exist a set LE $\bar{a}$ and sets $N_{1}$ and $N_{2}$ both of $\mu^{*}$-measure 0 such that $N_{1} \subset T^{\prime}, N_{2} \subset T, N_{2} \subset L^{\prime}$, and $\left(T \cap N_{2}^{\prime}\right) \cup N_{1}=L$.

By the definition of $\mu^{*}$, there exists a family $\left\{G_{n}\right\}_{n=1}^{\infty}$ of open sets such that $G_{n} \supset T$ and $\mu^{\prime}\left(G_{n}\right)-\frac{1}{n}<\mu^{*}(T)$. Writing $U=\bigcap_{n=1}^{\infty} G_{n}$, we clearly have $\mu^{*}(U)=\mu^{*}(T)$; and since $T$ is $\mu^{*}$-measurable, $\mu^{*}\left(U \cap T^{\prime}\right)=0$. By 5.5, $G_{n}=Q_{n} \cup S_{n}$, where $Q_{n}$ is the union of a countable family of compact sets and $\mu^{*}\left(S_{n}\right)=0$. Thus $U=\bigcap_{n=1}^{\infty} G_{n}$ $=\bigcap_{n=1}^{\infty}\left(Q_{n} \cup S_{n}\right)=\bigcap_{n=1}^{\infty} Q_{n} \cup M$, where $M \subset \bigcup_{n=1}^{\infty} S_{n}, \mu^{*}(M)=0$, and $\left(\bigcap_{n=1}^{\infty} Q_{n}\right) \cap M=0$. Writing $L=\bigcap_{n=1}^{\infty} Q_{n}$, we see that $L$ is in $\bar{a}$ and that $T \cup\left(U \cap T^{\prime}\right)=L \cup M$. Thus upon setting $T \cap M=N_{2}$ and $L \cap T^{\prime}=N_{1}$, we have $0=\mu^{*}(M)=\mu^{*}\left(N_{2}\right), 0=\mu^{*}\left(U \cap T^{\prime}\right)=\mu^{*}\left(N_{1}\right)$, and $\left(T \cap N_{2}^{\prime}\right) \cup N_{1}=L$. Since $N_{2} \subset M$, and $M \subset L^{\prime}$, it is clear that $N_{2} \subset L^{\prime}$.

§6. $\mu^{*}$-measurability and summability. The present section is devoted to showing the equivalence between $\mu^{*}$-integration and summing by the functional $M$.

6. 1 Theorem. Let $Q$ be a subset of $X$ such that $\mu^{*}(Q)=0$. Then ${ }_{Q}$ is sum- 
mable, and $\underline{\bar{M}}\left(\bullet_{Q}\right)=0$.

By the definition of $\mu^{*}$, for every $\varepsilon>0$, there exists an open set $G \supset Q$ such that $\mu^{\prime}(G)<\varepsilon$. Since $\iota_{G} \in \mathbb{R}$ and $\iota_{G} \triangleq \ell_{Q}$, it follows that $\bar{M}\left(\ell_{Q}\right) \leqq M_{\mathcal{L}}\left(\iota_{G}\right)=\mu^{\prime}(G)<\varepsilon$. Thus $\bar{M}\left(\iota_{Q}\right)=0$, and by $4.8, \underline{M}\left(\iota_{Q}\right)=0$ as well.

6.2 Theorem. Let $L$ be any set in the family $\bar{a}$ such that $\mu^{*}(L)$ is finite. Then $L$ is $\mu^{*}$-measuable, ${ }_{L} L$ is summable, and $\mu^{*}(L)=\underline{\bar{M}}\left(\ell_{L}\right)$.

It is proved by one of the present writers [8] that $L$ is $\mu^{*}$-measurable, and that for every $\varepsilon>0$, there exists a compact set $A$ such that $A \subset L$ and such that $\mu(A)+\varepsilon>\mu^{*}(L)$. We may now write the inequalities $\underline{M}\left(\ell_{L}\right) \geqq M_{\mathfrak{L}}\left(\iota_{A}\right)=\mu(A)$ $>\mu^{*}(L)-\varepsilon$, which are obvious. By 5.3 , we have $\mu^{*}(L)=\bar{M}\left(\iota_{L}\right)$, and thus $\underline{M}\left(\ell_{L}\right)$ $\geqslant \bar{M}\left(\iota_{L}\right)$. Theorem 4.8 shows that $\iota_{L}$ is actually summable and that $\underline{\bar{M}}\left(\iota_{L}\right)=\mu^{*}(L)$.

6. 3 Theorem. Let $T$ be any $\mu^{*}$-measurable subset of $X$ such that $\mu^{*}(T)<+\infty$. Then ${ }^{\prime} T$ is summable, and $\mu^{*}(T)=\underline{\bar{M}}\left({ }_{{ }_{T}}\right)$.

Let $N_{1}, N_{2}$, and $L$ be the sets described in 5.7. Then $T \cup N_{1}=L \cup N_{2}$, and since $T \cap N_{1}=L \cap N_{2}=0$, we have $\iota_{T}+\ell_{N_{1}}=\ell_{T} \cup N_{1}=\ell_{L} \cup N_{2}=\ell_{L}+\ell_{N_{2}}$. Using the easily verified inequality $\bar{M}\left(\iota_{T}+\iota_{N_{1}}\right) \leqq \bar{M}\left(\iota_{T}\right)+\bar{M}\left(\iota_{N_{1}}\right)$, and noting that $\iota_{L}$ and $\iota_{V_{2}}$ are summable, in view of 6.2 and 6.1 , we may write $\underline{\bar{M}}\left(\iota_{L}\right)=\underline{\bar{M}}\left(\ell_{L}\right)+\underline{\bar{M}}\left(\iota_{N_{2}}\right)=\underline{\bar{M}}\left(\iota_{L}\right.$ $\left.+\iota_{N_{2}}\right)=\bar{M}\left(\iota_{T}+\iota_{N_{1}}\right) \leqq \bar{M}\left(\iota_{T}\right)+\bar{M}\left(\iota_{N_{1}}\right)=\bar{M}\left(\iota_{T}\right) \leqq \bar{M}\left(\iota_{T}+\ell_{N_{1}}\right)$. (The last inequality follows from the monotonicity of $\bar{M}$.) Thus $\bar{M}\left(\ell_{T}\right)=\underline{\bar{M}}\left(\ell_{L}\right)$. We now observe that since $\mu^{*}\left(N_{i}\right)=0$, there exist open sets $G_{i}$ such that $G_{i} \supset N_{i}$ and $\mu^{\prime}\left(G_{i}\right)<\varepsilon(i=1,2)$. Using once again the compact set $A \subset L$ referred to in 6.2 , we deduce the following relations: $B=A \cap G_{1}^{\prime} \cap G_{2}^{\prime} C T ; B \in a ; \mu(B)>\mu(A)-2 \varepsilon>\mu^{*}(L)-3 \varepsilon$. Since ${ }_{{ }_{B}} \in \mathfrak{U}$, we have $\underline{M}\left(\iota_{T}\right) \equiv M_{\mathfrak{u}}\left(\iota_{B}\right)=\mu(B)>\mu^{*}(L)-3 \varepsilon$. From the relations $\mu^{*}(L)$ $=\underline{\bar{M}}\left(\iota_{L}\right)$ and $\underline{M}\left(\iota_{T}\right) \leqq \bar{M}\left(\iota_{T}\right)$, we thus infer that $\underline{M}\left(\iota_{T}\right)=\bar{M}\left(\iota_{T}\right)=\mu^{*}(L)=\mu^{*}(T)$.

6. 4 THEOREM. Let $T$ be any subset of $X$ such that ir $_{T}$ is summable. Then $T$ is $\mu^{*}$-measurable, and $\mu^{*}(T)=\bar{M}\left(\ell_{T}\right)$. Furthermore, $T$ is the union of a countable family of compact sets and a set of $\mu^{*}$-measure 0 .

By 5.3 , we have $\mu^{*}(T)=\underline{\underline{M}}\left(\iota_{T}\right)$. By 5.4 , there exists, for every positive integer $n$, a compact set $A_{n} \subset T$ such that $\mu\left(A_{n}\right)+\frac{1}{n}>\underline{M}\left(\iota_{T}\right)$. As in 5.5 , we see that $\mu^{*}\left(\bigcup_{n=1}^{\infty} A_{n}\right)=\underline{\bar{M}}\left(\iota_{T}\right)=\mu^{*}(T)$. Also as in 5.5 , we note that $\bigcup_{n=1}^{\infty} A_{n}$ is $\mu^{*}$-measurable and that $\mu^{*}\left(T \cap\left(\bigcup_{n=1}^{\infty} A_{n}\right)^{\prime}\right)=\mu^{*}(T)-\mu^{*}\left(\bigcup_{n=1}^{\infty} A_{n}\right)=0$. As the union of $\bigcup_{n=1}^{\infty} A_{n}$ and a set of $\mu^{*}$-measure $0, T$ is certainly $\mu^{*}$-measurable. Thus the present theorem is verified.

We remark in passing that 6.4 cannot be extended to the case of sets $T$ such that $\underline{M}\left(\iota_{T}\right)=+\infty$. For such $T$, it is clear that $\mu^{*}(T)=+\infty$; but $T$ need 
not be $\mu^{*}$-measurable. (For example, take $X=R, M(c)=\int_{-\infty}^{\infty} c(x) d x$, and $T=[0$, $\infty) \cup Q$, where $Q$ is a non-Lebesgue measurable subset of $(-1,0)$. Then $M\left(\iota_{T}\right)$ $=+\infty$, but $T$ is clearly not $\mu^{*}$-measurable.)

We now prove the equivalence of $\mu^{*}$-integration and summation by $\underline{\bar{M}}$ in the simplest case.

6.5 Theorem. Let $\varphi$ be a $\mu^{*}$-measurable function on $X$ such that $0 \leqq \varphi(x)$ $\leqq+\infty$ and such that $\int_{X} \varphi(x) d \mu^{*}(x)<+\infty$. Then $M(\varphi) \supseteqq \int_{X} \varphi(x) d \mu^{*}(x)$.

Using the definition of the integral given by Saks $[6$, p. 19], we have, for every $\varepsilon>0$, a finite sequence of $\mu^{*}$-measurable sets, $A_{1}, A_{2}, \ldots, A_{m}$, such that $A_{i} \cap A_{j}=0$ for $i \neq j, A_{1} \cup A_{2} \cup \ldots \cup A_{m}=E[x ; \varphi(x)>0], \mu^{*}\left(A_{i}\right)<+\infty$, and $\int_{X} \varphi(x) d \mu^{*}(x)<\sum_{i=1}^{m} \beta_{i} \mu^{*}\left(A_{i}\right)+\varepsilon$, where $\beta_{i}=\inf _{x \in A_{i}} \varphi(x)$. Consider the function $\omega(x)$ $=\sum_{i=1}^{m} \beta_{i} \iota_{A_{i}}(x)$. It follows from 6.3 and 4.11 that $\underline{\bar{M}}(\omega)=\sum_{i=1}^{m} \beta_{i} \mu^{*}\left(A_{i}\right)$. Since $\omega \leqq \varphi$, we have $\underline{M}(\varphi) \supseteqq \underline{\bar{M}}(\omega)=\underline{M}(\omega)=\sum_{i=1}^{m} \beta_{i} \mu^{*}\left(A_{i}\right)>\int_{X} \varphi(x) d \mu^{*}(x)-\varepsilon$, and these inequalites prove the present theorem.

6. 6 TheOREm. Let $\varphi$ be a bounded, non-negative, $\mu^{*}$-measurable function on $X$ such that $\mu^{*}(E[x ; \varphi(x)>0])$ is finite. Then $\bar{M}(\varphi) \leqq \int_{X} \varphi(x) d \mu^{*}(x)$.

Let $P=E[x ; \varphi(x)>0]$, let $\varepsilon$ be an arbitrary positive number, let $n$ be a positive integer $>\frac{\mu^{*}(P)}{\varepsilon}$, and let $J$ be the least integer $\triangleq\left[\sup _{\alpha \in X} \varphi(x)\right] \cdot n$. Let $P_{j}=E\left[x ; \frac{j-1}{n}<\varphi(x) \leqq \frac{j}{n}\right]$, for $j=1,2, \ldots, J$. Let us denote the function $\sum_{j=1}^{J} \frac{j-1}{n} \ell_{P_{j}}$ by $\omega_{1}$ and the function $\sum_{j=1}^{J} \frac{j}{n} \ell_{P_{j}}$ by $\omega_{2}$. The inequalities $\omega_{1} \leqq \varphi \leqq \omega_{2}$ are obvious. Furthermore, it is clear that $\omega_{1}$ and $\omega_{2}$ are $\mu^{*}$-measurable and that $\int_{X} \omega_{1}(x) d \mu^{*}(x)=\sum_{j=1}^{J} \frac{j-1}{n} \mu^{*}\left(P_{j}\right)$ and $\int_{X} \omega_{2}(x) d \mu^{*}(x)=\sum_{j=1}^{J} \frac{j}{n} \mu^{*}\left(P_{j}\right)$. Next, 6.4, 4.9, 4.10, and 4.11 imply that $\omega_{1}, \omega_{2}$, and $\omega_{2}-\omega_{1}$ are summable and that $\underline{\bar{M}}\left(\omega_{1}\right)=\sum_{j=1}^{j}{ }^{j-1} n$ $\mu^{*}\left(\boldsymbol{P}_{j}\right), \underline{\bar{M}}\left(\omega_{2}\right)=\sum_{j=1}^{J} \frac{j}{n} \mu^{*}\left(P_{j}\right)$, and $\underline{\bar{M}}\left(\omega_{2}-\omega_{1}\right)=\sum_{j=1}^{J} \frac{1}{n} \mu^{*}\left(P_{j}\right)$. Thus we have $\bar{M}\left(\omega_{2}\right.$ $\left.-\omega_{1}\right)=\underline{\bar{M}}\left(\omega_{2}\right)-\underline{\bar{M}}\left(\omega_{1}\right)$. The definition of $n$ and additivity of $\mu^{*}$ show that $\underline{\bar{M}}\left(\omega_{2}-\omega_{1}\right)=\frac{1}{n} \sum_{j=1}^{J} \mu^{*}\left(P_{j}\right)=\frac{1}{n} \mu^{*}(P)<\varepsilon$. It follows that $\underline{\bar{M}}\left(\omega_{1}\right)>\underline{M}\left(\omega_{2}\right)-\varepsilon$. The inequality $\omega_{1} \leqq \varphi$ implies that $\int_{X} \omega_{1}(x) d \mu^{*}(x) \leqq \int_{X} \varphi(x) d \mu^{*}(x)$, and the inequality $\varphi \leqq \omega_{2}$ implies that $\bar{M}(\varphi) \leqq \bar{M}\left(\omega_{2}\right)=\bar{M}\left(\omega_{2}\right)$. We combine the above relations, and have $\int_{X} \varphi(x) d \mu^{*}(x) \geqq \int_{X} \omega_{1}(x) d \mu^{*}(x)=\underline{\bar{M}}\left(\omega_{1}\right)>\underline{\bar{M}}\left(\omega_{2}\right)-\varepsilon=\bar{M}\left(\omega_{2}\right)-\varepsilon \geqq \bar{M}(\varphi)-\varepsilon$. 
This completes the present proof.

6. 7 Theorem. Let $\varphi$ be a function on $X$ such that $0 \leqq \varphi \leqq+\infty, \varphi$ is $\mu^{*}$ measurable, $\mu^{*}(E[x ; \varphi(x)>0])<+\infty$, and $\int_{X} \varphi(x) d \mu^{*}(x)<+\infty$. Then $\bar{M}(\varphi)$ $\leqq \int_{X} \varphi(x) d \mu^{*}(x)$.

Utilizing again the definition of integral set forth in Saks, loc. cit., we find that the sums $\sum_{k=1}^{n} k \mu^{*}(E[x ; k<\varphi(x) \leqq k+1])$ are all bounded above by $\int_{x} \varphi(x) d \mu^{*}(x)$. Thus the series $\sum_{k=1}^{\infty} k \mu^{*}(E[x ; k<\varphi(x) \leqq k+1])$ converges to a sum $\leqq \int_{X} \varphi(x) d \mu^{*}(x)$. I t follows immediately that $\lim _{m \rightarrow \infty} m \mu^{*}(E[x ; m<\varphi(x)])=0=\lim _{m \rightarrow \infty} \mu^{*}(E[x ; m<\varphi(x)])$. Let $\varepsilon$ be any positive number and let $p$ be a positive integer such that $\mu^{*}(E[x$; $p<\varphi(x)])<\varepsilon$. Define functions $\varphi_{p}$ and $\rho_{p}$ as follows :

$$
\begin{aligned}
& \varphi_{p}(x)=\left\{\begin{array}{cl}
\varphi(x) & \text { if } \varphi(x) \leqq p \\
0 & \text { if } \varphi(x)>p
\end{array} ;\right. \\
& \rho_{p}(x)=\varphi(x)-\varphi_{p}(x) .
\end{aligned}
$$

The function $\varphi_{D}$ clearly satisfies the hypotheses of 6.6, and therefore there exists a function $h \in \mathbb{Q}$ such that $h \geqq \varphi_{p}$ and $M_{\mathcal{Q}}(h)-\varepsilon \leqq \underline{M}\left(\varphi_{p}\right)=\int_{x} \varphi_{p}(x) d \mu^{*}(x)$. We now examine the function $\rho_{p}$, and for convenience we write $E[x ; k<\varphi(x) \leqq k+1]$ as $Z_{k}(k=p, p+1, \ldots)$. The sets $Z_{k}$ are certainly $\mu^{*}$-measurable and of finite $\mu^{*}$-measure. Let $G_{k}$ be an open set containing $Z_{k}$ such that $\mu^{\prime}\left(G_{k}\right)-\frac{\varepsilon}{2^{k}}<\mu^{*}\left(Z_{k}\right)$ $(k=p, p+1, \ldots)$. Let $g_{n}=\sum_{k=p}^{n}(k+1) \iota_{\sigma_{k}}(n=p, p+1, \ldots)$. It is clear that the functions $g_{n}$ are an increasing sequence of functions in $\mathfrak{L}$, that $g=\sum_{k=p}^{\infty}(k+1) \ell_{c_{i}}$ $=\lim _{n \rightarrow \infty} g_{n}$ is in $\mathfrak{L}$, and that $g \cong \rho_{p}$. We apply 4.15 and 6.4 to write $M_{\mathfrak{R}}(g)=\lim _{n \rightarrow \infty}$ $\left.M_{\mathfrak{L}}\left(g_{n}\right)=\lim _{n \rightarrow \infty}\left(\sum_{k=p}^{n}(k+1) \mu^{\prime} G_{k}\right)\right)=\sum_{k=p}^{\infty}(h+1) \mu^{\prime}\left(G_{k}\right)$. We apply inequalities set for th above to write $M_{\mathcal{L}}(g)=\sum_{k=p}^{\infty} k \mu^{\prime}\left(G_{k}\right)+\sum_{k=p^{\prime}}^{\infty} \mu^{\prime}\left(G_{k}\right)<\sum_{k=p}^{\infty} k\left(\mu^{*}\left(Z_{k}\right)+\frac{\varepsilon}{2^{k}}\right)+\sum_{k=p}^{\infty}\left(\mu^{*}\left(Z_{k}\right)+\frac{\varepsilon}{2^{k}}\right)$ $<\sum_{k=p}^{\infty} k \mu^{*}\left(Z_{k}\right)+\sum_{k=1}^{\infty} \mu^{*}\left(Z_{k}\right)+2 \varepsilon+\frac{\varepsilon}{2^{p-1}} \leqq \int_{x} \rho_{p}(x) d \mu^{*}(x)+3 \varepsilon+\frac{\varepsilon}{2^{p-1}}$. We combine these results with the inequality on $h$ set forth above and thereupon infer

$$
M_{\mathfrak{L}}(g+h)=M_{\mathfrak{L}}(g)+M_{\mathfrak{L}}(h)<\int_{X} \rho_{p}(x) d \mu^{*}(x)+\int_{X} \varphi_{p}(x) d \mu^{*}(x)+\varepsilon\left(4+\frac{1}{2^{p-1}}\right) .
$$

Since $g+h \supseteqq \varphi$ and since $\varepsilon$ is arbitrary, it follows that

$$
\bar{M}(\varphi) \leqq M_{\mathfrak{L}}(g+h) \leqq \int_{X} \rho_{p}(x) d \mu^{*}(x)+\int_{X} \varphi_{p}(x) d \mu^{*}(x)=\int_{X} \varphi(x) d \mu^{*}(x) .
$$

6. 8 Theorem. Let $\varphi$ be any function on $X$ such that $0 \leqq \varphi \leqq+\infty, \varphi$ is $\mu^{*}$ measurable, and $\int_{X} \varphi(x) d \mu^{*}(x)<+\infty$. Then $\bar{M}(\varphi) \leqq \int_{X} \varphi(x) d \mu^{*}(x)$. 
The present theorem is proved by an argument quite analogous to that used in 6.7. We define functions

$$
n \varphi(x)=\left\{\begin{array}{cc}
\varphi(x) & \text { if } \frac{1}{n} \leqq \varphi(x) \\
0 & \text { if } \varphi(x)<\frac{1}{n}
\end{array},\right.
$$

and $\sigma_{n}=\varphi-n \varphi$. We next write $Y_{k}=E\left[x ; \frac{1}{k+1} \leqq \varphi(x)<\frac{1}{k}\right]$. The series $\sum_{k=1}^{\infty} \frac{1}{k+1}$ $\mu^{*}\left(Y_{k}\right)$ and $\sum_{k=1}^{\infty} \frac{1}{k} \mu^{*}\left(Y_{k}\right)$ converge; the function $n \varphi$ satisfies the hypotheses of 6.7, for every positive integer $n$, and $\bar{M}\left(\sigma_{n}\right)$ is small. The remainder of the proof is modelled closely after that of 6.7 and is omitted.

We collect the results of 6.5 and 6.8 .

6.9 THEOREM. Let $\varphi$ be any function on $X$ such that $0 \leqq \varphi \leqq+\infty, \varphi$ is $\mu^{*-}$ measurable, and $\int_{X} \varphi(x) d_{\ell^{*}}(x)<+\infty$. Then $\varphi$ is summable, and $\bar{M}(\varphi)=\int_{X} \varphi(x)$ $\boldsymbol{d}_{\mu}^{*}(x)$.

The converse of 6.9 is also true.

6.10 Theorem. Let $\varphi$ be a function on $X$ such that $0 \leqq \varphi \leqq+\infty$ and $\varphi$ is summable. Then $\varphi$ is $\mu^{*}$-measurable and $\underline{\bar{M}}(\varphi)=\int_{x} \varphi(x) d \mu^{*}(x)$.

For every positive integer $n$, there exist functions $f_{n} \in \mathbb{U}$ and $g_{n} \in \mathbb{Q}$ such that $f_{n} \leqq \varphi \leqq g_{n}$ and $M_{\mathfrak{U}}\left(f_{n}\right)+\frac{1}{n}>\underline{\bar{M}}(\varphi)>M_{\mathcal{L}}\left(g_{n}\right)-\frac{1}{n}$. We may suppose that $f_{n} \leqq f_{n+1}$ and $g_{n+1} \leqq g_{n}(n=1,2, \ldots)$. Since $f_{n}$ and $g_{n}$ are $\mu^{*}$-measurable, 6.9 implies that $M_{\mathfrak{l}}\left(f_{n}\right)=\int_{X} f_{n}(x) d \mu^{*}(x)$ and $M_{\mathcal{L}}\left(g_{n}\right)=\int_{X} g_{n}(x) d \mu^{*}(x)$. Writing $\phi=\lim _{n \rightarrow \infty} f_{n}$ and $\omega=\lim _{n \rightarrow \infty} g_{n}$, we have, clearly enough, $\psi \leqq \varphi \leqq \omega$. Lebesgue's theorem on term-by-term integration $\left[6\right.$, p. 29] shows that $\int_{X} \psi(x) d \mu^{*}(x)=\int_{X} \omega(x) d \mu^{*}(x)$ $=\bar{M}(\varphi)$. It follows that $\varphi-\psi$ is a function vanishing except on a set of $\mu^{*}$ measure 0 ; acoordingly $\varphi$ is $\mu^{*}$-measurable and $\bar{M}(\varphi)=\int_{X} \omega(x) d \mu^{*}(x)=\int_{X} \varphi(x)$ $d \mu^{*}(x)$.

\section{BIBLIOGRAPHY}

[1] Banach, Stefan. Théorie des opérations linéaires, Monografje Matematyczne, Warszawa, 1932.

[2] Bourbaki, Nicolas. Éléments de mathématique, Livre III, ch. III et IV. Hermann et Cie., Paris, 1942.

[3] Bourbaki, Nicolas. Éléments de mathématique, Livre III, ch. IX. Hermann et Cie., Paris, 1948. 
[4] Cartan, Henri. Sur les fondements de la théorie du potentiel. Bull. Soc. Math. France, 69, pp. 71-96 (1941).

[5] Halmos, Paul R. Measure theory. D. Van Nostrand and Co., New York, 1950.

[6] Saks, Stanislas. Theory of the integral. Monografje Matematyczne, Warszawa-Lwow, 1937.

[7] Stone, M. H. Applications of the theory of Boolean rings to general topology. Trans. Amer. Math. Soc., 41, pp. 375-481 (1937).

[8] Hewitt, Edwin. Integration in locally compact spaces I, To appear in Univ. of Washington Studies in Mathematics.

University of Washington

Seattle, Washington, U.S.A. 